\title{
Ermittlung eines Skelettierungsverfahrens zur Konvertierung von Topologieoptimierungsergebnissen
}

\author{
Investigation of surface skeletonization methods for reconstruction of topology \\ optimized structures
}

\author{
Johannes Mayer ${ }^{1 *}$, Sandro Wartzack ${ }^{1}$ \\ ${ }^{1}$ Engineering Design, Friedrich-Alexander-Universität Erlangen-Nürnberg \\ ${ }^{*}$ Korrespondierender Autor: \\ Johannes Mayer \\ KTmfk - Lehrstuhl für Konstruktionstechnik \\ Martensstraße 9 \\ 91058 Erlangen \\ Telefon: +49 (0) 9131/8523215 \\ Mail: mayer@mfk.fau.de
}

\begin{abstract}
By showing where to place material in a specified design space, the continuous topology optimization is very useful in product development of structural loaded components. However the optimizations design proposal is far from being a parametric CAD-model. Instead it usually consists of a tesselated surface, that has to be re-engineered. As this is often done manually, it takes a lot of time and expert knowledge. Curve skeleton algorithms have proven their ability to support the restructuring of truss-like design proposals by providing references for construction elements. To investigate a similar design concept for general optimization results, showing truss-like structures as well as surface-like sections, the suitability of surface skeletons for topology reconstruction is examined.
\end{abstract}

\section{Keywords}

Computational Geometry, Topology Optimization, Medial Axis Transform, Surface Skeleton 


\section{Automatische Geometrieerzeugung als übergeordnetes Ziel}

Die kontinuierliche Topologieoptimierung mit der SIMP-Methode (solid isotropic material penalization) ist hervorragend zum Entwurf beanspruchungsgerechter Leichtbaustrukturen geeignet. In dieser Methode werden die Elementdichten eines Finite-Elemente-Modells zwischen null (entspricht einem Bereich ohne Material) und eins (entspricht einem Bereich mit Material) variiert. Für einen gegebenen Lastfall wird damit eine kraftflussgerechte Materialverteilung innerhalb eines definierten Bauraums ermittelt. Allerdings resultiert aus einer Optimierung mit dem SIMP-Verfahren kein fertiges Bauteil, sondern lediglich eine elementweise Untergliederung des Bauraums mit Bereichen unterschiedlicher Dichte. [1] Dadurch hat die repräsentierte Struktur keine klar definierte Berandung und ist weder parametrisch, noch anderweitig referenziert. Um zu einer parametrischen, featurebasierten CAD-Geometrie zu gelangen, ist zunächst eine Interpretation der resultierenden Materialdichteverteilung in Material- und Nicht-Materialbereich notwendig. Dadurch wird eine abstrakte, mit Dreiecken tesselierte Oberfläche erzeugt. Anschließend muss durch CADEntwurf mit gleichzeitiger Beachtung der Gerechtheiten des „Design for X“ (DfX) die geometrische Bauteilgestalt rekonstruiert werden (vgl. Bild 1) [2].

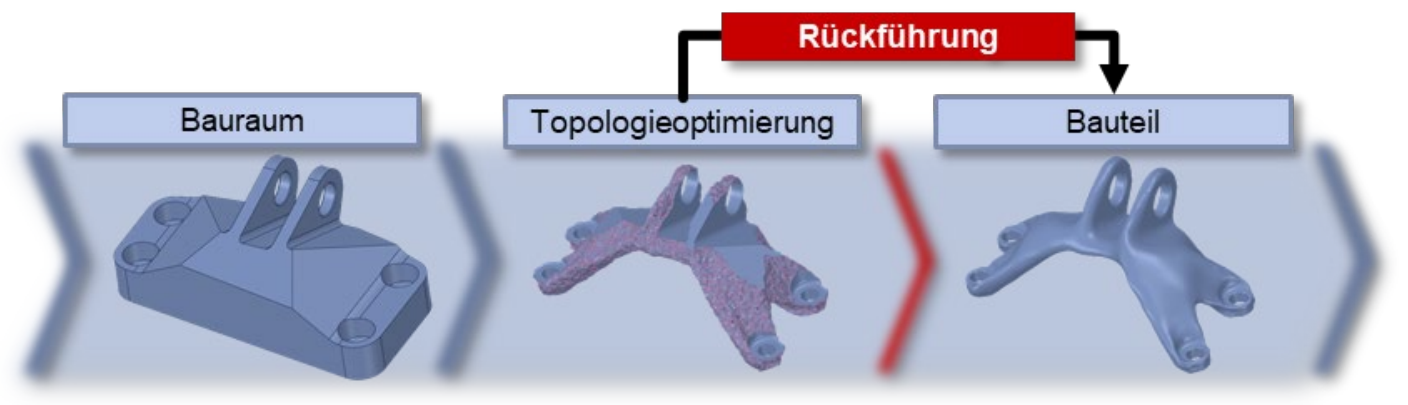

Bild 1: Grundlegende Schritte zur Topologieoptimierung eines Strukturbauteils im Produktentwicklungsprozess am Beispiel einer praktischen Optimierungsaufgabe [3].

Die Rückführung erfolgt zumeist manuell, wodurch die Integration der Methode in den Produktentwicklungsprozess durch hohe Anforderungen an Expertenwissen und benötigter Zeit erschwert wird. Eine durchgängig automatisierte Prozesskette bietet an dieser Stelle Vorteile. Dabei soll die Geometrie parametrisch-assoziativ gestaltet sein, damit sie in der Produktentwicklung auch nach dem Schritt der Topologieoptimierung verändert werden kann. Denn statt der unreferenzierten Repräsentation der Geometrie durch ein abstraktes Oberflächennetz, entspricht vielmehr die Geometrieanpassung und Variantenbildung durch Zugriff auf Parameter den Anforderungen der Ingenieurinnen und Ingenieure in der Produktentwicklung. Zur Automatisierung der Geometrierückführung ist es daher das übergeordnete Ziel, beliebige Topologien in parametrische Bauteile zu rekonstruieren.

\section{Bestehende Ansätze zur Geometrierückführung in einem durchgängigen Prozess}

Bestehende Ansätze der Geometrierückführung können in flächen- und volumenorientierte Verfahren klassifiziert werden. Bei flächenorientierten Verfahren wird die Strategie verfolgt, ein aus der Optimierung resultierendes Oberflächennetz in eine der Endkontur entsprechende Gestalt zu bringen.

Dieser Ansatz wird von KOGUCHI und KIKUCHI [4], TANG und CHANG [5] sowie HsU und Hsu [6] verfolgt. Im Ansatz von Hsu und Hsu [6] werden Querschnitte des Optimierungsergebnisses durch dreidimensionale B-Spline-Kurven approximiert und anschließend durch Zug-Konstruktionselemente zu einer glatten Oberfläche verbunden. In ähnlicher Weise werden bei TANG und CHANG [5] Querschnitte durch kubische B-Splines 
erzeugt und in der Umgebung des CAD-Programms zu Hüllgeometrie rekonstruiert. Vor der Querschnittapproximation wird hier eine Glättung des Oberflächennetzes durchgeführt [5]. KOGUCHI und KIKUCHI [4] klassifizieren jeden Knoten des Oberflächennetzes entweder als Eckpunkt oder als einer Kante bzw. einer Fläche zugehörig und approximieren anschließend die Flächenbereiche durch biquartische B-Splines. Auch von HESSEL [7] wird eine Bereichsunterteilung mit jeweils einer Annäherung durch NURBS-Freiformflächen (NonUniformal Rational B-Spline) vorgenommen.

Innerhalb der kommerziell vertriebenen Programme solidThinking Inspire [8] sowie 3DExperience Functional Generative Designer [9] der Firmen Altair und Dassault wird ebenfalls auf die Rückführung in Oberflächengeometrie zurückgegriffen, wobei innerhalb von solidThinking Inspire [8] wiederum auf die Annäherung des Oberflächennetzes durch NURBSFlächen zurückgegriffen wird.

Die derart rekonstruierte Bauteilgeometrie wird lediglich durch die Oberfläche als Berandung definiert. Wie dargelegt, beruht diese diese Strategie vielfach auf der Approximation des Oberflächennetzes durch Freiformflächen, weshalb eine Geometrievariation im Sinne der Anforderungen eines Produktentwicklers durch einfachen Zugriff auf einzelne Parameter nicht vorgenommen werden kann.

Neben Hilfsmitteln zur flächenorientierten Rückführung bietet die Software 3-matic [10] von Materialise auch die Möglichkeit zur Erkennung von Mittellinien des Oberflächenetzes und darauf basierender Rekonstruktion von CAD-Features wie beispielsweise eines ZugKonstruktionselements. Zwar ist dabei auch ein Nutzereingriff erforderlich, doch die erzeugte Geometrie kann in diesem Vorgehen mit Parametern versehen werden und wird von Volumina gebildet.

Speziell für die Geometrierückführung von Topologieoptimierungsergebnissen entwickelte, volumenorienterte Ansätze sind die Methoden von LARSEN und JENSEN [11], STANGL [12] und CUILLIĖRE [13]. In der Vorgehensweise nach LARSEN und JENSEN [11] wird vom Bauraum schrittweise Material durch vordefinierte Features wie dem Konstruktionselement Extrusion entfernt. Die Vorgaben für diese bool'schen Operationen werden anhand der Hauptkoordinatenachsen für jede Raumrichtung erzeugt. Als Resultat lassen sich innerhalb dieses Ansatzes keine Strukturmerkmale erfassen, die nicht parallel zu den Hauptachsen liegen. Die rein abtragende Modellierungsweise schafft darüberhinaus keine Parametrik, mit der direkt auf die Konstruktionsgeometrie referenziert werden kann.

Anders verhält es sich mit den skelettbasierten Ansätzen von STANGL [12] und CUILLIĖRE [13]. AUCH KRESSLEIN [14] nutzt zur Abstraktion des ursprünglichen Oberflächennetzes die vereinfachte Darstellungsform eines Kurvenskeletts. Als solches wird eine eindimensionale Kurve bezeichnet, die im Wesentlichen innerhalb einer dreidimensionalen Form liegt und deren topologische Gestalt wiedergibt [14]. Das Kurvenskelett dient sowohl bei STANGL [12], wie auch bei CUILLIĖRE [13] als Referenz für ZugKonstruktionselemente, die zusammen mit definierten Querschnitten Volumenmodelle bilden. Beide Ansätze werden jeweils an stabförmigen Geometrien vorgestellt, wobei CUILLIĖRE [13] sein Vorgehen explizit auf Formen dieser Art bezieht. Er beschreibt Schwierigkeiten im Verlauf des Kurvenskeletts an Stellen, an denen mehrere Stäbe aufeinandertreffen [13]. KRESSLEIN [14] wertet in seiner Methode Querschnitte an orthogonal zum Skelett liegenden Ebene aus und kommt dabei u.a. zu dem Ergebnis, dass diese Strategie gerade bei Geometrien, an denen meherere stabförmige Bereiche aufeinandertreffen oder bei flächigen Geometrien an Grenzen stößt.

\section{Flächenskelette als mögliche Grundlage zur Geometrierückführung}

Wie CUILLIĖRE [13] herausstellt, existieren neben Kurvenskeletten auch verschiedene Verfahren, um sogenannte Flächenskelette zu ermitteln. Während eindimensionale Kurvenskelette die topologische Gestalt von Fachwerkstrukturen sehr gut erfassen [15], 
erscheint zur Rekonstruktion von Topologien mit plattenartigen Bereichen die zweidimensionale Ausprägung der Flächenskelette geeignet. Diese Verfahren wurden nicht für den Anwendungszweck der Topologierückführung entwickelt und ihre Ergebnisse unterscheiden sich zudem in der Art ihrer geometrischen Informationen [13, 15]. Aus diesen Gründen stellt sich die Frage nach der Auswahl einer Skelettbeschreibung für die Geometrierückführung:

Welches Flächenskelett ist für die Rekonstruktion von Ergebnissen der Topologieoptimierung geeignet?

Zur Untersuchung geeigneter Abstrahierungsmöglichkeiten einer Geometrie werden die Ergebnisse zweier Verfahren gegenübergestellt. Das Skelett, das im Mean Curvature FlowVerfahren erzeugt wird, ist nicht in einer theoretisch formalisierten Basis definiert [13, 16]. Dagegen entspricht die Mittelachsentransformation als zweite hier betrachtete Skelettbeschreibung der Definition eines Flächenskeletts nach BLUM [17]. Demnach handelt es sich dabei um die Vereinigung aller Mittelpunkte der größten einbeschriebenen Kugeln einer Struktur, was als Mediale Achse bezeichnet wird [17].

\subsection{Die Skelettbeschreibung durch das Mean Curvature Flow-Verfahren}

Der Skelettierungsalgorithmus Mean Curvature Flow erzeugt durch iterative Kontraktion des Oberflächennetzes ein Kurvenskelett. Jeder Knoten erfährt dabei abhängig von der lokalen Krümmung eine antinormal gerichtete Verschiebung. Die als Mesoskelett bezeichneten Zwischenergebnisse bilden eine Mischtopologie, die neben Kurven- auch Flächenbereiche enthält. [16]
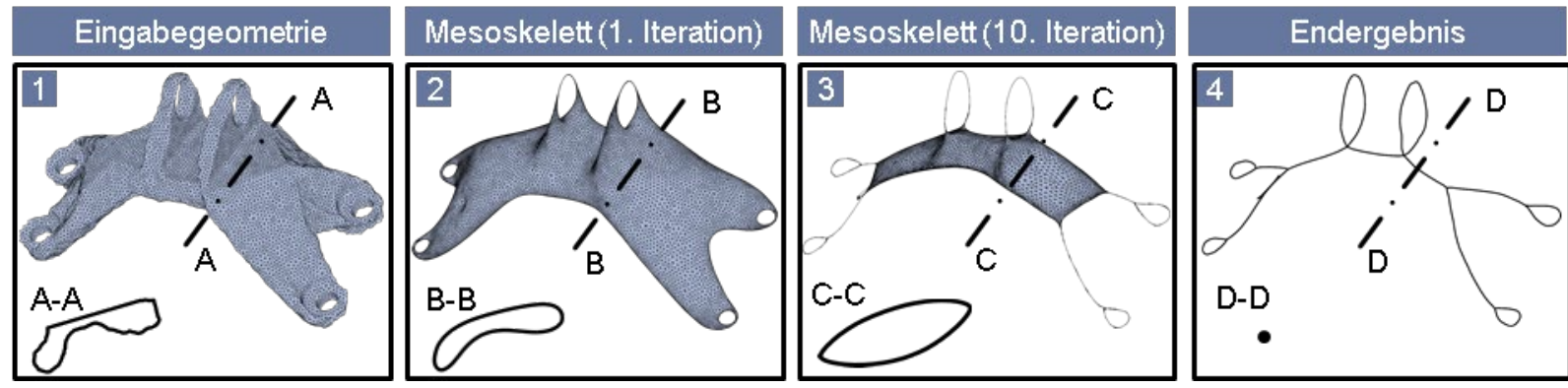

Bild 2: Schematischer Ablauf der Skelettkonvertierung durch Mean Curvature Flow mit lokalem Querschnitt [18]

Da zur Beschreibung der Optimierungsergebnisse nicht das letztendlich resultierende Kurvenskelett, sondern ein Mesoskelett verwendet werden soll, stellt sich die Frage, bei welchem Iterationsschritt des Verfahrens das entsprechende Ergebnis für die Weiterverwendung zur Topologierückführung vorliegt. Prinzipbedingt umschließen die Flächenbereiche der Mesoskelette Volumen (vgl. Querschnitte Bild 2). Mit iterativ fortschreitender Kontraktion werden Netzkanten, die unterhalb einer definierten Kantenlänge liegen, kollabiert und die Vernetzung angepasst. Neben der Netzabhängigkeit besteht eine Abhängigkeit von Parametern, die das Kontraktionsverhalten in Geschwindigkeit und dem Grad der Anpassung an die Mediale Achse steuern. Die Zwischenergebnisse wie auch das Endergebnis sind von der Vernetzung abhängig (vgl. Bild 5). [16]

\subsection{Die Skelettbeschreibung durch die Mittelachsentransformation}

Bei der Medialen Achse handelt es sich um ein Flächenskelett nach BLUMS Definition [17]. Durch die Ermittlung der größten einbeschriebenen Kugeln ist der Abstand eines 
Skelettknotens zur Strukturberandung in Form der Kreisradien bekannt. Die Radien ergeben in Kombination mit der Medialen Achse die Mittelachsentransformation. Der Definition zufolge handelt es sich hierbei um eine kontinuierliche Struktur, die in der hier zugrundeliegenden Implementierung mittels Voronoi-Diagrammen approximiert wird. [19]

Weil das als Eingabedatei verwendete Oberflächennetz diskret verteilte Knotenpunkte aufweist, ist die ermittelte Mittelachsentransformation hier ebenfalls eine diskret verteilte Information. Der Begriff soll daher im Folgenden auch die diskrete Form einschließen.
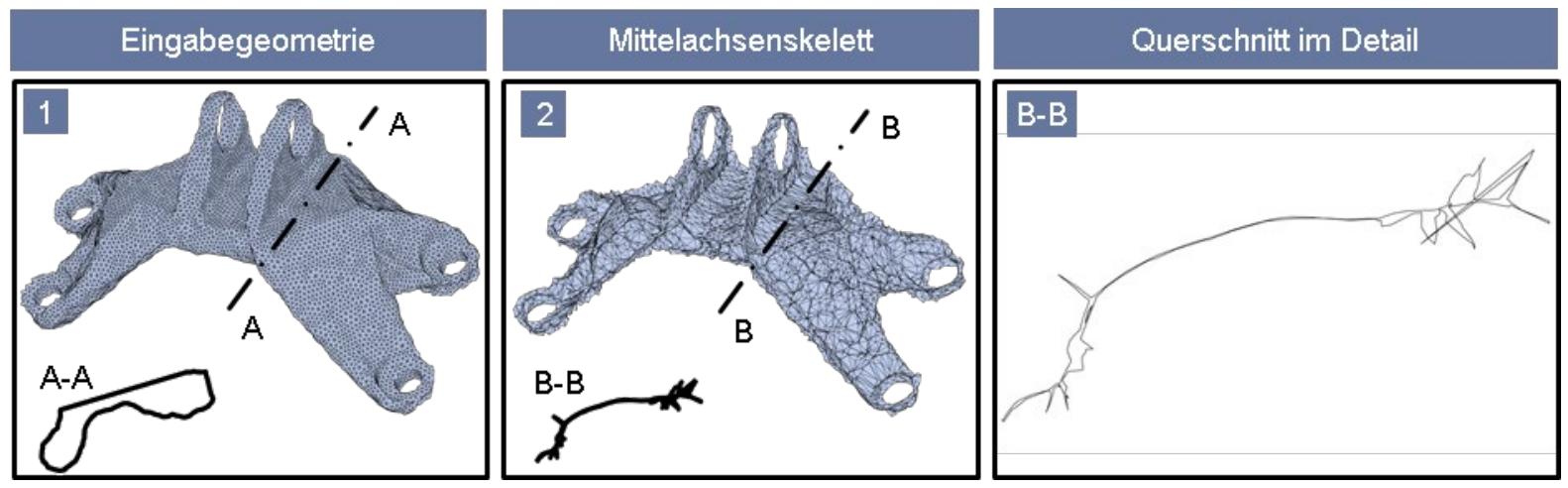

Bild 3: Eingangsgeometrie und resultierendes Mittelachsenskelett mit lokalem Querschnitt

Die Konnektivität der Punkte auf der Medialen Achse richtet sich nach dem eingangs verwendeten Oberflächennetz [19]. Da ein Skelettpunkt jedoch prinzipbedingt mindestens zwei zugehörige Punkte auf der Oberfläche hat, ergibt sich eine Konnektivität, die nicht frei von Überschneidungen ist. Außerdem werden kleine Oberflächenunstetigkeiten von der Medialen Achse berücksichtigt und spiegeln sich in ausgeprägten Kanten oder Facetten wieder. Dies ist vor allem bei detaillierter Betrachtung eines Skelettquerschnittes ersichtlich (vgl. Bild 3).

Da der Algorithmus parameterunabhängig ist, resultiert für dieselbe Geometrie stets dasselbe Flächenskelett. Abweichungen stellen sich hierbei lediglich aufgrund der Diskretisierung ein. Aus einer Oberflächenvernetzung mit größerer Kantenlänge folgt demnach ein Flächenskelett mit ebenfalls größerer Kantenlänge. Für kleinere Diskretisierung nähert sich die Mittelachsentransformation an die theoretische Mediale Achse an. [19]

\section{Gegenüberstellung der Algorithmen Mean Curvature Flow und Mittelachsentransformation hinsichtlich der Rückführung von Konstruktionsgeometrie}

Da der Vergleich von Skelettierungverfahren zumeist qualitativ erfolgt und diese im Kontext der Topologierückführung in einen anderen Zusammenhang gebracht werden, erfolgt die anschließende Bewertung auf Basis von Kriterien, die durch Expertenwissen bezüglich der speziellen Anforderungen im Rahmen der Topologierückführung festgelegt wurden [15].

Der Vergleich des Mean Curvature Flow-Verfahrens und der Mittelachsentransformation wird in Tabelle 1 übersichtlich anhand einiger Kriterien dargestellt. Diese Kriterien sollen die Eignung des jeweiligen Verfahrens zur Rückführung von Konstruktionsgeometrie im direkten Vergleich feststellen. Sie resultieren aus dem Expertenwissen, das aus der praktischen Anwendung der Topologieoptimierung mit der SIMP-Methode und der Rekonstruktion von Geometrie stammt. Im Folgenden werden die einzelnen Aspekte näher beleuchtet. 
Tab. 1: Vergleich der Skelettdarstellungen nach Kriterien zur Rückführbarkeit in Konstruktionsgeometrie

\begin{tabular}{lcc}
\hline Bewertungskriterien (-/0/+) & Mittelachsenskelett & Mesoskelett \\
\hline Anforderungen an Eingabedaten & 0 & 0 \\
Anwendbarkeit bei beliebigen Geometrien & + & 0 \\
Reproduzierbarkeit des Ergebnisses & + & 0 \\
Eindeutige Flächenrepräsentation & 0 & - \\
Verknüpfung mit Eingabedaten & + & - \\
Reproduzierbarkeit der Eingabedaten & 0 & - \\
Netzabhängigkeit & 0 & - \\
Erkennung regelmäßiger Geometrien & - & - \\
\hline Gesamt & +2 & -5 \\
\hline
\end{tabular}

\subsection{Anforderungen an Eingabedaten}

Im STL-Datenformat, das oftmals für Optimierungsergebnisse herangezogen wird, besteht Geometrie aus einer Vielzahl zusammengesetzter Dreiecksfacetten. Diese Facetten bilden insgesamt ein Oberflächennetz, wobei die Konnektivität aller Knotenpunkte festgelegt ist. Durch den Normalenvektor der Dreiecke ist der Innen- und Außenbereich der Oberfläche definiert.

Der Mean Curvature Flow-Algorithmus verlangt ein Oberflächennetz als Eingabeformat [16]. Die Mittelachsentransformation ist prinzipiell mit einer Punktewolke durchführbar, stellt jedoch Anforderungen an die Dichte und Art der Punkteverteilung, die einer $C^{1}$-stetigen Oberfläche entsprechen muss [19].

\subsection{Anwendbarkeit bei beliebigen Geometrien}

Sofern die Anforderungen an das Datenformat erfültt sind, ist mit beiden Verfahren eine Skelettkonvertierung durchführbar. Jedoch kann es beim Mean Curvature Flow-Verfahren dazu kommen, dass das Skelett die Bauraumgrenzen überschreitet (vgl. Querschnitte Bild 2).

Aus der Topologieoptimierung mit der SIMP-Methode resultiert auch ein Bereich, für den explizit kein Material vorgesehen werden sollte. Für die Rekonstruktion ist es interessant, auch diesen komplementären Bereich per Skelettkonvertierung zu abstrahieren. Damit können innerhalb der automatisierten Rückführung beispielsweise subtraktive Modellierungsschritte nachempfunden werden. Verlässt das Mesoskelett die von der Eingangsgeometrie vorgegebenen Grenzen, ist zumindest eine gesonderte Untersuchung an diesen Stellen erforderlich. Dahingegen liegt das Flächenskelett der Mittelachsentransformation vollständig im Inneren des verwendeten Oberflächennetzes (vgl. Querschnitte Bild 3).

\subsection{Reproduzierbarkeit des Ergebnisses}

Vorausgesetzt die Eingangsgeometrie ist identisch, bleibt die Form des Flächenskeletts bei erneuter Skelettkonvertierung mit der Mittelachsentransformation unverändert. Bei der Verwendung des Mean Curvature Flow-Algorithmus muss dazu der Iterationsschritt bekannt sein, an dem das Mesoskelett extrahiert wurde. Zudem müssen die Parameter identisch sein, um dasselbe Ergebnis zu erhalten. Damit ist das resultierende Flächenskelett mit diesem Verfahren schwieriger zu reproduzieren. 


\subsection{Eindeutige Flächenrepräsentation}

Die topologische Gestalt des zu abstrahierenden Oberflächennetzes wird vom Mesoskelett teils durch Flächen repräsentiert, die ihrerseits wiederum Volumen einschließen (vgl. Bild 2 und Bild 5). Daduch wird die Geometrie, von der das Mesoskelett abgeleitet wurde, nicht ausschließlich durch die Flächen des Mesoskeletts beschrieben, sondern durch das von den Flächen eingefasste Volumen.

Die Abgrenzung von fachwerkähnlichen und flächigen Strukturbereichen kann anhand der Kurven- und Flächenbereiche des Mesoskeletts getroffen werden. Wo das Skelett zu einer Kurve kontrahiert ist, handelt es sich um einen als Fachwerk zu interpretierenden Bereich, während die Flächenbereiche des Mesoskeletts auf flächige Strukturbereiche der Eingangsgeometrie verweisen. Es stellt sich dabei die Frage, bis zu welchem Grad das Mesoskelett in Richtung des Kurvenskeletts kontrahiert werden soll bzw. zu welchem Zeitpunkt des Iterationsverfahrens die Skelettkonvertierung abgeschlossen ist. Damit ist die Interpretation fachwerkähnlicher und gedrungener Strukturbereiche nicht eindeutig.

Das Mittelachsenskelett schließt hingegen kein Volumen ein und approximiert die Eingabedaten stets in gleicher Weise (vgl. Bild 3 und Bild 5). Die Ausprägung des Skeletts lässt eine Unterteilung flächiger und nicht flächiger Geometriebereiche prinzipiell zu. So ist die Mediale Achse eines langen idealen Zylinders im Wesentlichen eine Linie, wohingegen Formen mit abweichendem Querschnittsverhältnis von Länge zu Breite als Flächen dargestellt werden. In Bereichen topologieoptimierter Geometrie ist keine geometrisch ideale Grundform zu erwarten, sodass in der Skelettrepräsentation keine Abschnitte in Kurvenform vorliegen. Unstetigkeiten in der Oberfläche bewirken darüber hinaus eine ausgeprägte Beeinflussung des Skeletts. Gerade bei Topologieoptimierungsergebnissen mit der meist ungeradlinig verlaufenden Berandung ist dies häufig der Fall. Zudem ist die Konnektivität des Mittelachsenskeletts nicht überschneidungsfrei und nicht wasserdicht (vgl. Querschnitt Bild 3).

\subsection{Verknüpfung mit Eingabedaten}

Im Gegensatz zum Kontraktionsverfahren ist der Abstand des Mittelachsenskeletts vom Oberflächennetz im Ergebnis der Mittelachsentransformation enthalten. Zum einen ist damit eine definierte Verbindung zwischen den Eingangsdaten und dem konvertierten Skelett herstellbar. Zum anderen ermöglicht die Auswertung des Radius eine lokal aufgelöste Betrachtung der Strukturstärke. Das heißt, durch die Mittelachsentransformation kann der Durchmesser der Struktur an jedem Ort analysiert werden, auch wenn die geometrische Form nicht einer Standardgeometrie wie einem Zylinder o.ä. entspricht (vgl. Bild 4).

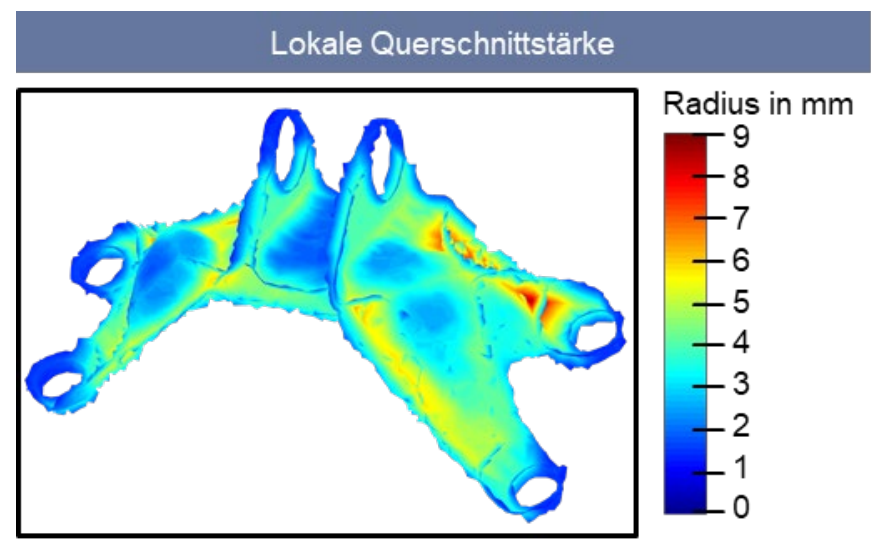

Bild 4: Graphisch dargestellte Querschnittstärke am Skelett der Mittelachsentransformation 
Die geometrische Information lokaler Querschnittstärke ist im Kontext der Geometrierückführung von hoher Relevanz, weil sie als grundlegende Maßangabe für verschiedene Konstruktionselemente herangezogen werden kann.

\subsection{Reproduzierbarkeit der Eingabedaten}

Vom Skelett auf die eingangs verwendete optimierte Struktur zu schließen, erhöht die Transparenz im Umgang mit dem Skelett als Datenform. So könnte für eine Datenweitergabe allein die Skelettbeschreibung ausreichen oder selbst nach der Geometrierückführung noch auf das unbearbeitete Optimierungsergebnis rückgeschlossen werden.

Zwar ist für eine gegebene Geometrie die Mediale Achse eindeutig, im Umkehrschluss gilt dies jedoch nicht. Mit Hinzunahme der Kreisradien ist eindeutig auf die Geometrie zu schließen. Die hier vorliegende diskrete Mittelachsentransformation lässt bedingt und näherungsweise auf die Eingangsgeometrie schließen. Das Mesoskelett ist nicht in die Eingangsgeometrie zurückzuwandeln.

\subsection{Netzabhängigkeit}

Für einen visuellen Vergleich des Netzeinflusses wurde eine beispielhafte Geometrie einer isotropen Vernetzung mit den Kantenlängen $2 \mathrm{~mm}$ und $5 \mathrm{~mm}$ unterzogen. Für diese unterschiedlichen Oberflächennetze wurde mit beiden Verfahren ein Flächenskelett erzeugt.
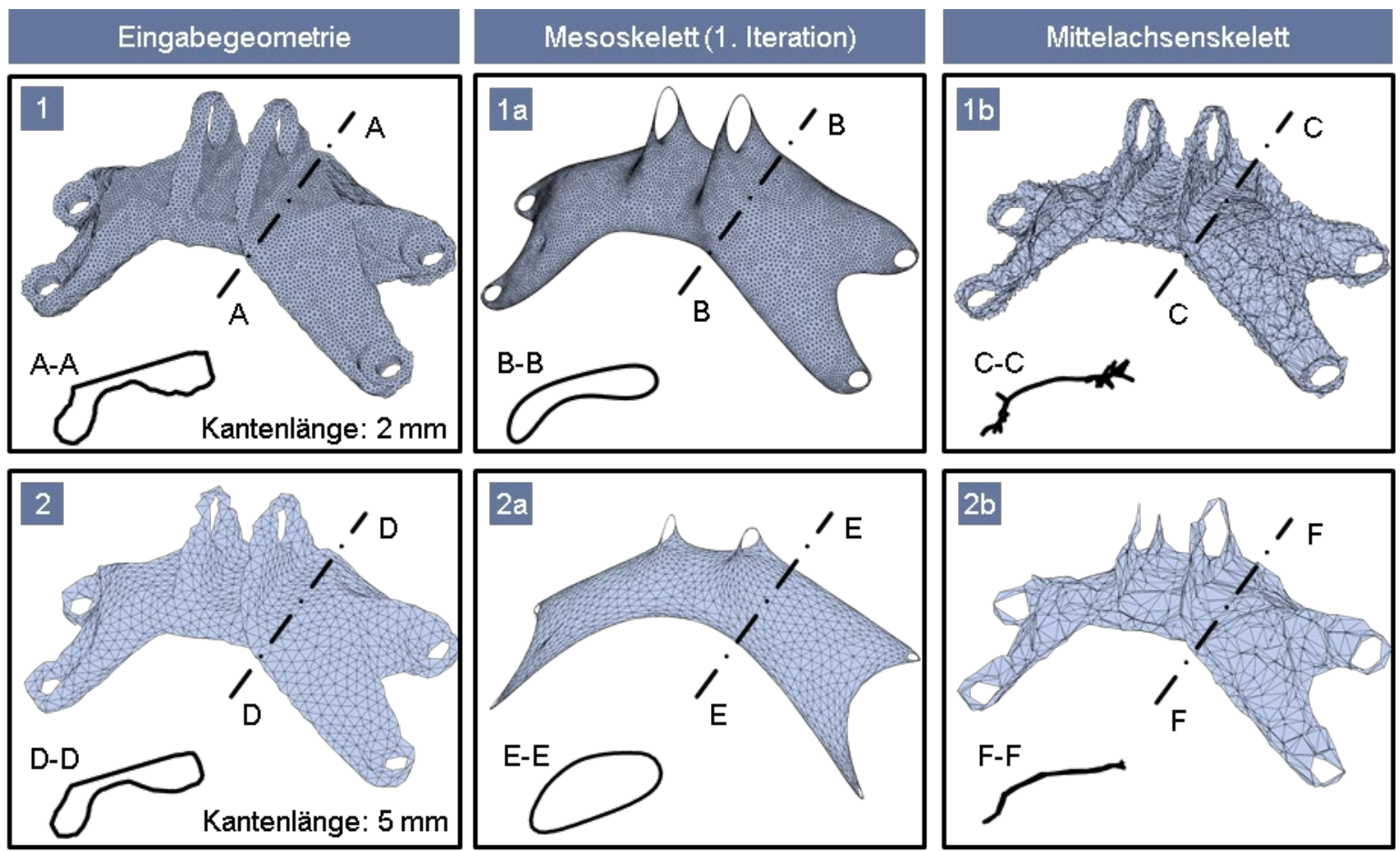

Bild 5: Vergleich der Auswirkung unterschiedlicher Netzkantenlängen auf die Flächenskelette [18]

Beide hier verglichenen Verfahren werden von der Diskretisierung beeinflusst. Es ist erkennbar, dass die Netzkantenlänge von $5 \mathrm{~mm}$ in diesem Beispiel eine ungünstig große Auflösung darstellt, da eine Bolzenlasche im Mittelachsenskelett nicht mehr vollständig abgebildet wird (vgl. Bild 5). Beim Mean Curvature Flow-Algorithmus ist insgesamt eine qualitativ stärkere Abweichung zwischen den Skeletten unterschiedlicher Netzkantenlänge vorhanden. Das Mittelachsenskelett strebt hingegen bei variierender Vernetzung in unterschiedlicher Genauigkeit gegen die theoretische Mediale Achse (vgl. Bild 5). 


\subsection{Erkennung regelmäßiger Geometrien}

Weder mit dem Meso- noch mit dem Mittelachsenskelett ist es ohne Weiteres möglich, geometrisch regelmäßige Formen in der Eingangsgeometrie als solche zu identifizieren. Diese Erkennung ist nützlich, um Strukturbereiche des Topologieoptimierungsergebnisses zu selektieren, die nicht Teil der Materialdichtevariation waren. Vorab aus der Optimierung ausgeschlossene Bereiche sollten in unbeeinflusster Form im Endergebnis der Rekonstruktion enthalten sein.

\subsection{Auswertung der Gegenüberstellung von Mean Curvature Flow und Mittelachsentransformation}

Der Vergleich von Skelettierungsalgorithmen ist aufgrund fehlender gemeinsamer Formalismen nicht quantifizierbar. Einzig die Berechnungsdauer des Skelettes wäre hier denkbar, doch diese blieb unberücksichtigt, weil der Zeitanspruch für die computergestütze Berechnung beider Verfahren gegenüber der manuellen Bauteilrekonstruktion als vergleichsweise gering eingeschätzt wird. Bei der Bewertung wurde stattdessen Wert auf die Tauglichkeit zur Rekonstruktion von Geometrie gelegt.

Die Aufteilung einer Topologie in Kurven- und Flächenbereiche gestaltet sich anhand von Mesoskeletten schwierig, da das Mesoskelett als Zwischenergebnis direkt von der Wahl des Unterbrechungszeitpunktes abhängt. Zudem besteht in der Parameterangabe zur Steuerung der Kontraktionsgeschwindigkeit ein weiterer Einflussfaktor. Die Mittelachsentransformation repräsentiert fachwerkartige Bereiche in der Praxis durch Flächen statt Kurven. Jedoch unterscheiden sich diese durch kleinere Flächen und kleinere Kreisradien im Vergleich zur Reststruktur flächiger Bereiche.

An der Auswertung in Tabelle 1 wird deutlich, dass das Verfahren der Mittelachsentransformation als vorteilhafter für den gedachten Anwendungszweck eingeschätzt wird. Mit diesem Verfahren ist es möglich, ein Optimierungsergebnis in reproduzierbarer Weise zu abstrahieren. Dabei verletzt die Skelettform nicht den vorgegebenen Bauraum und beinhaltet u.a. durch die Radien der größten einbeschriebenen Kugeln lokale Querschnittinformation.

Die Rekonstruktion technischer Bauteile erfordert je nach Gestalt viele Informationen wie zum Beispiel geometrische Längenangaben. Um den Rekonstruktionsprozess allgemeiner Strukturbauteile vollständig zu automatisieren, wird daher die höhere Informationsdichte des Mittelachsenskeletts als Vorzug bewertet.

Dennoch ist es nicht möglich, aus der Mittelachsentransformation ohne weitere Schritte ein endkonturnahes CAD-Bauteil abzuleiten. Vielmehr muss auf dieser Skelettkonvertierung als Dekomposition eine generalisierte Beschreibung der Topologie erarbeitet werden.

\section{Rückführung parametrischer, fertigungs- und beanspruchungsgerechter Konstruktionsgeometrie}

Ausgehend von der Idee, Ergebnisse der Topologieoptimierung durch einen skelettbasierten Ansatz zu abstrahieren, wurden zwei repräsentative Skelettierungsalgorithmen hinsichtlich einer Verwendung zur Geometrierekonstruktion verglichen. Die Bewertung erfolgte unter besonderer Berücksichtigung flächiger Geometrie. Das Verfahren der Mittelachsentransformation erfasst derartige Strukturbereiche ebenso wie auch fachwerkartige Strukturen. Außerdem ist es durch Querschnittstärkenermittlung und Parameterunabhängigkeit für die automatisierte Geometrierückführung geeignet. Nächste Schritte bestehen in der Entwicklung einer generalisierten Beschreibung der Topologie aufbauend auf der Mittelachsentransformation. Darauf basierend soll die automatisierte Geometriesynthese unter Berücksichtigung von DfX-Gerechtheiten mit dem Endresultat eines parametrischen, fertigungs- und beanspruchungsgerechten Bauteils erfolgen. 


\section{Danksagung}

Die vorgestellte Forschungsarbeit ist Teil des Forschungsprojekts WA 2913/36-1 „TopoRestruct - Rückführung fertigungs-, beanspruchungs- und funktionsgerechter Konstruktionsgeometrie aus Ergebnissen der Topologieoptimierung in den Produktentwicklungsprozess" und gefördert durch die Deutsche Forschungsgemeinschaft (DFG). Die Autoren danken der Deutschen Forschungsgemeinschaft (DFG) für die finanzielle Unterstützung.

Für den Inhalt dieser Publikation sind die Autoren verantwortlich.

\section{Literaturverzeichnis}

[1] Bendsøe, Martin P.; Sigmund, Ole: Topology Optimization - Theory, Methods and Applications. Berlin: Springer, 2004.

[2] Pahl, Gerhard; et al.: Konstruktionslehre. Grundlagen erfolgreicher Produktentwicklung. Methoden und Anwendung. Berlin: Springer, 2007.

[3] General Electric; Grabcad.: GE jet engine bracket challenge. GrabCAD. URL https://grabcad.com/challenges/ge-jet-engine-bracket-challenge. - abgerufen am 2019-10-30.

[4] Koguchi, Atsushi; Kikuchi, Noburu: A surface reconstruction algorithm for topology optimization. Engineering with Computers. Vol. 22 (2006), Nr. 1, pp. 1-10.

[5] Tang, Poh-Soong; Chang, Kuang-Hua: Integration of topology and shape optimization for design of structural components. Structural and Multidisciplinary Optimization. Vol. 22 (2001), Nr. 1, pp. 65-82.

[6] Hsu, Ming-Hsiu; Hsu, Yeh-Liang: Interpreting three-dimensional structural topology optimization results. Computers \& structures. Vol. 83 (2005), Nr. 4, pp. 327-337.

[7] Hessel, Christoph: Integration der Topologieoptimierung in den CAD-gestützten Entwicklungsprozess. Aachen, Shaker, 2003.

[8] Singh, Himanshu; et al.: Topology Optimization \& Casting Process Simulation. Whitepaper, Altair Engineering Inc. 2017.

[9] Dassault Systèmes: 3DExperience Catia Release Highlights - R2018x. Product brochure, Dassault Systèmes, 2018.

[10] Blanc, Christophe: 3-matic STL 9.0. Von der Topologieoptimierung zum gedruckten Bauteil, Materialise beschreitet neue Wege. 11. Coachulting Forum, 11.03.2015.

[11] Larsen, Shane; Jensen, Greg: Converting Topology Optimization Results into Parametric CAD Models. Computer Aided Design and Applications. Vol. 6 (2009), Nr. 3, pp. 407-418.

[12] Stangl, Thomas; Wartzack, Sandro: Feature based interpretation and reconstruction of structural topology optimization results. In: Weber, C.; Husung, S.; Cascini, G.; Cantamessa, M.; Marjanovic, D.; Graziosi, S. (Eds.): Proceedings of the 20th International Conference on Engineering Design (ICED15). Vol. 6 (2015). 27.-30.07.2015, Milan, Italy. Design Society, 2015, pp. 235-244.

[13] Cuillière, Jean-Christophe; François, Vincent; Nana, Alexandre: Automatic construction of structural CAD models from 3D topology optimization. In: Computer Aided Design and Applications Vol. 15 (2017), Nr. 1, pp. 107-121.

[14] Kresslein, Jacob; et al.: Automated cross-sectional shape recovery of 3D branching structures from point cloud. In: Journal of Computational Design and Engineering. Vol. 5 (2018), pp. 368-378.

[15] Tagliasacchi, Andrea; et al.: 3D Skeletons: A State-of-the-Art Report. In: Madeira, J.; Patow, G.; Romão, T. (eds.): Computer Graphics Forum. Vol. 35 (2016), Nr. 2, pp. 573-597.

[16] Tagliasacchi, Andrea; et al.: Mean curvature skeletons. Computer Graphics Forum Vol. 31 (2012), Nr. 5, pp. $1735-1744$.

[17] Blum, Harry: A Transformation for Extracting New Descriptors of Shape. In: Wathen-Dunn, Weiant (ed.): Models for the Perception of Speech and Visual Form. MIT Press, 1967. pp. 362-380.

[18] Gao, Xiang; Loriot, Sébastien; Tagliasacchi, Andrea: Triangulated Surface Mesh Skeletonization. In CGAL User and Reference Manual. CGAL Editorial Board, 5.0.2 edition, 2020.

[19] Amenta, Nina; Choi, Sunghee; Kolluri, Ravi Krishna: The Power Crust. In: Proceedings of the 6th ACM Symposium on Solid Modeling and Applications. New York, NY, USA: ACM, 2001, pp. 249-266. 